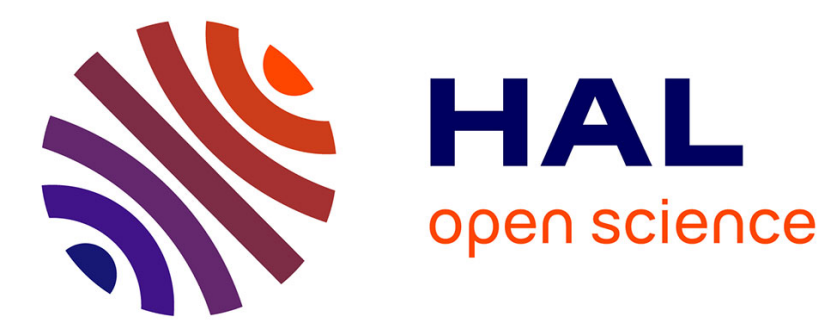

\title{
Rotating magnetohydrodynamic turbulence
}

N Bell, Sergey Nazarenko

\section{- To cite this version:}

N Bell, Sergey Nazarenko. Rotating magnetohydrodynamic turbulence. Journal of Physics A: Mathematical and Theoretical, 2019, 52 (44), pp.445501. 10.1088/1751-8121/ab44fb . hal-02386764

\section{HAL Id: hal-02386764 \\ https://hal.science/hal-02386764}

Submitted on 17 Dec 2020

HAL is a multi-disciplinary open access archive for the deposit and dissemination of scientific research documents, whether they are published or not. The documents may come from teaching and research institutions in France or abroad, or from public or private research centers.
L'archive ouverte pluridisciplinaire HAL, est destinée au dépôt et à la diffusion de documents scientifiques de niveau recherche, publiés ou non, émanant des établissements d'enseignement et de recherche français ou étrangers, des laboratoires publics ou privés. 
PAPER

\section{Rotating magnetohydrodynamic turbulence}

To cite this article: N K Bell and S V Nazarenko 2019 J. Phys. A: Math. Theor. 52445501

\section{Recent citations}

Constraints on ion versus electron heating by plasma turbulence at low beta

A. A. Schekochihin et al

View the article online for updates and enhancements.

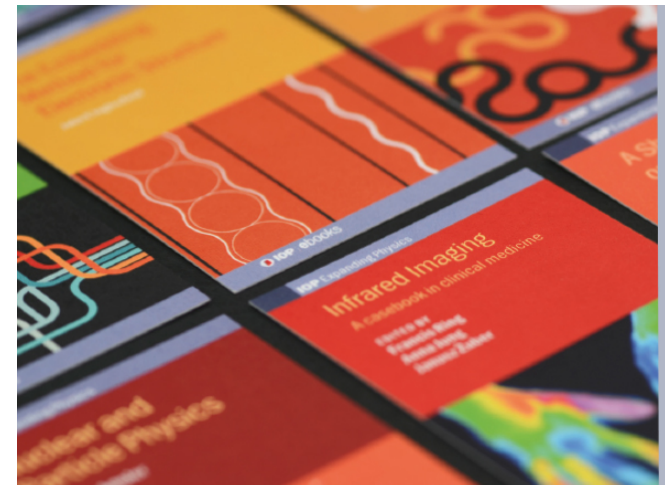

IOP ebooks

Bringing together innovative digital publishing with leading authors from the global scientific community. Start exploring the collection-download the first chapter of every title for free.

This content was downloaded from IP address 92.94 .170 .138 on 17/12/2020 at 13:21 


\title{
Rotating magnetohydrodynamic turbulence
}

\author{
N K Bell ${ }^{1}$ (i) and S V Nazarenko ${ }^{2,3}$ (우 \\ ${ }^{1}$ Mathematical Institute, University of Warwick, Coventry, CV4 7AL, \\ United Kingdom \\ 2 Institut de Physique de Nice, Université Nice-Sophia Antipolis, Parc Valrose, \\ 06108 Nice, France \\ E-mail: Sergey.Nazarenko@unice.fr
}

Received 15 May 2019, revised 22 August 2019

Accepted for publication 16 September 2019

Published 9 October 2019

\begin{abstract}
Decaying turbulence in rotating Magneto-hydrodynamic systems is studied theoretically and numerically. In the linear limit, when the velocity and magnetic perturbations are small, the system supports two types of waves. When the rotation effects are stronger than the ones of the external magnetic field, one of these waves contains most of the kinetic energy (inertial wave) and the other-most of the magnetic energy (magnetostrophic wave). The weak wave turbulence (WWT) theory for decoupled inertial and magnetospheric wave systems was previously derived in Galtier (2014 J. Fluid Mech. 757 114-54). In the present paper, we suggest theory of strong turbulence for such waves based on the critical balance (CB) approach conjecturing that the linear and nonlinear timescales are of similar magnitudes in a wide range of turbulent scales. Regimes of weak and strong wave turbulence are simulated numerically. The results appear to be in good agreement with the WWT and $\mathrm{CB}$ predictions, particularly for the exponents of the kinetic and magnetic energy spectra.
\end{abstract}

Keywords: MHD turbulence, rotating turbulece, wave turbulence

(Some figures may appear in colour only in the online journal)

\section{Introduction}

Astrophysical flows are generally electrically conducting prompting the use of magnetohydrodynamics (MHD) for describing their dynamics. Such flows are often accompanied by large-scale magnetic fields which results in the existence of a rich zoo of wave modes. This has motivated the use of weak wave turbulence (WWT) theory when studying MHD systems [2-6]. WWT theory describes the long-time statistical behaviour of weakly nonlinear

\footnotetext{
${ }^{3}$ Author to whom any correspondence should be addressed.
} 
dispersive waves $[7,8]$. In most physical situations however, there is a coexistence of strongly nonlinear coherent structures and weakly nonlinear waves. WWT theory is applicable when the timescale of the linear waves is much shorter than the nonlinear timescale. In strong wave turbulence, there may exist a regime where these two timescales are of the same order over a wide range of scales. This is the so-called critical balance $(\mathrm{CB})$ conjecture which was introduced in the context of MHD in [9].

Along with the presence of a mean magnetic field, astrophysical flows often undergo rotation about an axis. Rotating MHD then has wide application including planetary flows, stellar flows and accretion discs [10-12]. Often, fluid motions in such rotating planetary and astrophysical objects are in a turbulent state in characterised by presence of propagating waves and vortices which affect the mean dynamical characteristics and transport properties of matter and energy, in particular the rate of accretion, the rate of conversion of the kinetic energy into heat and dynamo action of generation of large-scale mean magnetic field. An interesting new mechanism of preferential alignment of the large-scale planetary and stellar magnetic field and rotation based on the nonlinearly interacting waves in rotating MHD was recently put forward in [13]. In this paper, we will consider weakly and strongly nonlinear regimes of rotating MHD turbulence assuming that the motions are strongly subsonic and sub-Alfvenic. We aim at predicting and numerically simulating an idealised system with a view that the basics properties in this system are more widely shared by turbulence in the above applications. Namely, an incompressible MHD system under solid body rotation and in the presence of a uniform background magnetic field will be considered. The governing equations in the rotating frame of reference are:

$$
\begin{aligned}
& \frac{\partial \boldsymbol{u}}{\partial t}+2 \Omega_{\mathbf{0}} \times \boldsymbol{u}+\boldsymbol{u} \cdot \nabla \boldsymbol{u}=-\nabla P_{*}+\boldsymbol{b}_{\mathbf{0}} \cdot \nabla \boldsymbol{b}+\boldsymbol{b} \cdot \nabla \boldsymbol{b}+\nu \nabla^{2} \boldsymbol{u} \\
& \frac{\partial \boldsymbol{b}}{\partial t}+\boldsymbol{u} \cdot \boldsymbol{\nabla} \boldsymbol{b}=\boldsymbol{b}_{\mathbf{0}} \cdot \boldsymbol{\nabla} \boldsymbol{u}+\boldsymbol{b} \cdot \nabla \boldsymbol{u}+\eta \boldsymbol{\nabla}^{2} \boldsymbol{b} \\
& \nabla \cdot \boldsymbol{u}=0 \\
& \nabla \cdot \boldsymbol{b}=0
\end{aligned}
$$

where $v$ is the velocity, $P_{*}$ is the total pressure, $\boldsymbol{b}$ is the magnetic field normalised to a velocity, $\boldsymbol{b}_{\mathbf{0}}$ is the uniform normalised magnetic field, $\Omega_{0}$ is the rotation rate and $\nu$ and $\eta$ are the kinematic viscosity and magnetic diffusivity respectively. For the remainder of this paper we shall assume that the axis of rotation is aligned with the background magnetic field,

$$
\Omega_{\mathbf{0}}=\Omega_{0} \hat{\boldsymbol{e}}_{\|}, \quad \boldsymbol{b}_{\mathbf{0}}=b_{0} \hat{\boldsymbol{e}}_{\|},
$$

where $\hat{\boldsymbol{e}}_{\|}$is a unit vector.

The addition of the Coriolis force from rotation yields dynamical effects the importance of which is measured by the Rossby number,

$$
\text { Ro }=\frac{U_{0}}{L_{0} \Omega_{0}},
$$

where $U_{0}, L_{0}$ and $\Omega_{0}$ are typical velocity, length scale and rotation rate respectively. The Rossby number is the ratio of the advection term and the Coriolis force in the Navier-Stokes equations. Small Rossby numbers thus correspond to flows in which the rotation is of significant importance as is the case for planetary flows especially in the case of large planets $[14,15]$. 
In rotating MHD there is also another important parameter-the magneto-inertial length,

$$
d=\frac{b_{0}}{\Omega_{0}} .
$$

The value of $d$ determines the relative strength of the Lorentz force and the Coriolis force. We find for the ratio of these two forces:

$$
\mathcal{D}=\frac{\left|\boldsymbol{b}_{\mathbf{0}} \cdot \nabla \boldsymbol{b}\right|}{\left|2 \boldsymbol{\Omega}_{\mathbf{0}} \times \boldsymbol{u}\right|} \sim \frac{b}{u} \frac{d}{l}
$$

When $\mathcal{D}$ is large, the Lorentz force is dominant and when it is small the Coriolis force is dominant. Assuming that $u$ and $b$ are known, the value of $\mathcal{D}$ can be determined using $d / l$, or equivalently, $k d$ in Fourier space.

There have been various numerical studies of the effects of rotation in hydrodynamic turbulence, for example [16-18]. The theoretical groundwork for WWT regime of the rotating turbulence has been developed in [19]. A prediction for the energy spectrum $E(k) \sim k_{\perp}^{-5 / 2}$ was found which applies when the waves in the rotating fluid (inertial waves) are weakly nonlinear. A prediction for strong turbulence based on $\mathrm{CB}$ was found in [6] - it gives the energy spectrum $E(k) \sim k_{\perp}^{-5 / 3}$. More recently a WWT theory has been developed for rotating MHD [1]. Predictions were made for the energy spectra in two asymptotic regions: $k d=k b_{0} / \Omega_{0} \rightarrow \infty$ and $k d \rightarrow 0$. As $k d \rightarrow \infty$, the linear waves collapse onto the Alfvén waves resulting in a $k^{-2}$ spectrum as first found in [2]. In the $k d \rightarrow 0$ limit, the left polarised waves (inertial waves) and the right polarised waves (magnetostrophic waves) become separate such that the inertial waves contain most of the kinetic energy and the magnetostrophic waves contain most of the magnetic energy. Thus, when the two types of waves are decoupled, the kinetic energy spectrum is therefore the same as for rotating hydrodynamic turbulence. Incidentially, the magnetic energy spectrum was also found to be $E(k) \sim k_{\perp}^{-5 / 2}$.

In this paper, we present numerical simulations of rotating MHD turbulence. We begin with an overview of the WWT theory and extend it to make a prediction of the energy spectra based upon the $\mathrm{CB}$ phenomenology. We then present two numerical simulations which aim to test the theoretical predictions.

\section{Wave modes in rotating MHD}

Linear waves in rotating MHD are circularly polarized and dispersive. The general solution for the frequency is given by

$$
\omega \equiv \omega_{\Lambda}^{s}=\frac{s k_{\|} \Omega_{0}}{k}\left(-s \Lambda+\sqrt{1+k^{2} d^{2}}\right),
$$

where $s= \pm 1$ defines the directional polarity such that we always have $s k_{\|}>0$ and $\Lambda= \pm s$ gives the circular polarity with $\Lambda=s$ indicating right polarization and $\Lambda=-s$ left polarization. The linear waves can be considered in the limits $k d \rightarrow \infty$ and $k d \rightarrow 0$. In the small scale limit $(k d \rightarrow \infty)$, the frequency of the right and left polarized waves collapse onto the Alfvén wave frequency. In this limit the turbulence properties can therefore be studied as Alfvén wave turbulence for which there exists a large body of work, for example [2, 6, 9, 20, 21]. In the large scale limit $(k d \rightarrow 0)$, the right and left polarized waves are the pure magnetostrophic waves and the pure inertial waves respectively. The frequencies are given by 


$$
\begin{aligned}
& \omega_{\mathrm{M}} \equiv \omega_{s}^{s}=\frac{s k_{\|} k \mathrm{~d} b_{0}}{2}, \\
& \omega_{\mathrm{I}} \equiv \omega_{-s}^{s}=\frac{2 s \Omega_{0} k_{\|}}{k} .
\end{aligned}
$$

For the magnetostrophic branch one can typically assume a balance between the Coriolis and Lorentz forces [11]. Following this assumption, the nonlinear evolution of the magnetic field under relatively strong rotation and uniform magnetic field can be described by the magnetostrophic equation [1]

$$
\frac{\partial \boldsymbol{b}}{\partial t}=-\frac{d}{2} \boldsymbol{\nabla} \times\left[(\boldsymbol{\nabla} \times \boldsymbol{b}) \times\left(\boldsymbol{b}+\boldsymbol{b}_{\mathbf{0}}\right)\right]+\eta \boldsymbol{\nabla}^{2} \boldsymbol{b} .
$$

This equation will be used to study the wave turbulence properties for the magnetostrophic waves. Note, however, that the balance between the Coriolis and Lorentz forces may break at the level of the nonlinear terms if the scales of the inertial and magnetostrophic waves are separated. In this case the two types of waves get coupled (see the second part of the appendix).

\section{Weak wave turbulence}

\subsection{Energy cascade spectra}

The WWT theory for rotating MHD was developed in [1]. The derivation will not be reproduced here but the results which provide context to our numerical simulations will be discussed. We turn our attention to the large scale limit $k d \rightarrow 0$. In this limit and assuming that nonlinear interactions occur locally in $k$-space it can be shown that in the leading order the inertial waves decouple from the magnetostrophic waves. This is because no resonance triads simultaneously containing both types of waves exist in this regime. A discussion of this decoupling and identification of a coupled regime can be found in the appendix to this paper. Furthermore in this limit, the inertial waves contain all of the kinetic energy and the magnetostrophic waves contain all of the magnetic energy. Both the inertial wave turbulence and the magnetostrophic wave turbulence are found to become anisotropic such that $k_{\perp} \gg k_{\|}$.

Let us first consider the inertial waves. The WWT theory applies when the dynamics are dominated by the weakly nonlinear waves. In terms of time scales this implies that the period of the linear waves is much shorter than the nonlinear turnover time,

$$
\tau_{\mathrm{I}} \ll \tau_{\mathrm{nl}} \text {. }
$$

Looking first at the momentum equation (1), the nonlinear time scale is

$$
\tau_{\mathrm{nl}} \sim \frac{1}{k \widetilde{U}}
$$

where $\widetilde{U}$ is the oscillating velocity, and the period of the inertial waves is

$$
\tau_{\mathrm{I}} \sim\left(\omega_{\mathrm{I}}\right)^{-1}=\frac{k}{2 s \Omega_{0} k_{\|}} .
$$

For a phenomenological derivation of the energy spectrum, we require the characteristic transfer time. If we assume that after a number of stochastic wavepacket collisions the cumulative effect may be regarded as a random walk, then we can use [22, 23]

$$
\tau_{\text {tr }} \sim \frac{\tau_{\text {nl }}^{2}}{\tau_{\mathrm{I}}}
$$


Assuming a stationary state in which the kinetic energy flux per unit mass $\left(\epsilon^{u}\right)$ is independent of scale we find

$$
\epsilon^{u} \sim \frac{E^{u}}{\tau_{\mathrm{tr}}} \sim \frac{E^{u}\left(k_{\perp}, k_{\|}\right) k_{\perp} k_{\|}}{\tau_{\mathrm{tr}}} .
$$

Then making use of $\widetilde{U}^{2} \sim E\left(k_{\perp}, k_{\|}\right) k_{\perp} k_{\|}$and the anisotropic assumption $k_{\perp} \gg k_{\|}$we have, after some algebra [19],

$$
E^{u}\left(k_{\perp}, k_{\|}\right) \sim \sqrt{\epsilon^{u} \Omega_{0}} k_{\perp}^{-5 / 2} k_{\|}^{-1 / 2} .
$$

To study the magnetostrophic waves, we look at the magnetostrophic equation (12). The nonlinear time scale is

$$
\tau_{\mathrm{nl}} \sim \frac{1}{k_{\perp}^{2} \mathrm{~d} \widetilde{B}}
$$

with $\widetilde{B}$ being the oscillating magnetic field, and the period of magnetostrophic waves is given by

$$
\tau_{\mathrm{M}} \sim\left(\omega_{\mathrm{M}}\right)^{-1}=\frac{2}{s k_{\|} k \mathrm{~d} b_{0}} .
$$

Performing the same analysis as for the kinetic energy spectrum, we find the magnetic energy spectrum [1]:

$$
E^{b}\left(k_{\perp}, k_{\|}\right) \sim \sqrt{\frac{\epsilon^{b} b_{0}}{d}} k_{\perp}^{-5 / 2}\left|k_{\|}\right|^{-1 / 2} .
$$

The same phenomenology can be applied to the other inviscid invariant of rotating MHD, the hybrid helicity [1]. Following the dual cascade argument, one can conclude that the hybrid helicity should cascade toward smaller $k$ 's (inverse cascade) whereas the energy-toward larger $k$ 's (direct cascade). The hybrid helicity cascade is an interesting topic for future study with possible insight into a dynamo process. In the present paper, we consider the direct energy cascade only.

\subsection{Domain of validity for weak wave turbulence approach}

WWT theory relies upon the time scale separation between the linear time scale (wave period) and the nonlinear time scale, i.e.

$$
\frac{\tau_{l}}{\tau_{\mathrm{nl}}} \ll 1
$$

In rotating MHD, there are two linear wave branches with different dispersion relations. We have defined different nonlinear times for each of these branches, given by equation (14) for the inertial waves and equation (19) for the magnetostrophic waves. For the respective ratios of these times we have:

$$
\begin{gathered}
\chi^{u}=\frac{\tau_{I}}{\tau_{\mathrm{nl}}^{u}} \sim \frac{k_{\perp}^{2} \widetilde{U}}{k_{\|} \Omega_{0}}, \\
\chi^{b}=\frac{\tau_{\mathrm{M}}}{\tau_{\mathrm{nl}}^{b}} \sim \frac{k_{\perp} \widetilde{B}}{k_{\|} b_{0}} .
\end{gathered}
$$


For the WWT theory to apply, the following two relations must hold: $\chi^{u} \ll 1$ and $\chi^{b} \ll 1$. It is clear that the ratios will vary with the wavenumber and thus may not remain uniformly small across the entire wavenumber range. The dependence of both $\chi^{u}$ and $\chi^{b}$ can be estimated using the WWT predictions along with $\widetilde{U} \sim \sqrt{E^{u}\left(k_{\perp}, k_{\|}\right) k_{\perp} k_{\|}}$and $\widetilde{B} \sim \sqrt{E^{b}\left(k_{\perp}, k_{\|}\right) k_{\perp} k_{\|}}$. One then finds

$$
\begin{aligned}
& \chi^{u} \sim\left(\frac{\sqrt{\epsilon^{u}}}{\Omega_{0}}\right)^{1 / 2} k_{\|}^{-3 / 4} k_{\perp}^{5 / 4}, \\
& \chi^{b} \sim\left(\frac{\sqrt{\epsilon^{b}}}{b_{0} d}\right)^{1 / 2} k_{\|}^{-3 / 4} k_{\perp}^{1 / 4} .
\end{aligned}
$$

Both $\chi^{u}$ and $\chi^{b}$ grow as $k_{\perp}$ increases and so there will be some scale (provided that dissipation is weak enough) at which the WWT assumption is broken and where the CB assumption becomes relevant. Such a transition from weak to strong wave turbulence has been observed in numerical simulations of Alfvén wave turbulence [24] and Hall MHD turbulence [25].

\subsection{Strong wave turbulence}

In strong wave turbulence, it is natural to assume that the energy spectrum saturates when the nonlinear interaction time becomes of the same order as the linear wave period over a wide range of turbulent scales [8]. Such states are known as a CB as introduced in MHD turbulence in [9]. It has been proposed that CB provides a universal scaling conjecture for determining the spectra of strong turbulence in anisotropic wave systems [26].

In the classical Kolmogorov spectrum of turbulence [27], the system is isotropic and highly nonlinear such that the transfer time is simply the nonlinear timescale. When waves are present there is an additional time scale, the period of the linear waves. The CB assumption

$$
\tau_{\mathrm{tr}} \sim \tau_{l} \sim \tau_{\mathrm{nl}}
$$

provides the additional scaling required to perform a heuristic derivation of the energy spectrum.

To derive the kinetic energy spectrum in the $\mathrm{CB}$ regime we shall again turn our attention to the momentum equation. The nonlinear time scale and the linear wave period are again given by (14) and (15) respectively. Balancing these time scales as per (27) gives

$$
\widetilde{U} \sim \frac{\Omega_{0} k_{\|}}{k_{\perp}^{2}}
$$

The kinetic energy flux is then found to be

$$
\epsilon^{u} \sim \frac{\widetilde{U}^{2}}{\tau_{\mathrm{tr}}} \sim \widetilde{U}^{3} k_{\perp} \sim \frac{\Omega_{0}^{3} k_{\|}^{3}}{k_{\perp}^{5}},
$$

which, when rearranged, gives the wavenumber scaling for the CB [26],

$$
k_{\|} \sim\left(\epsilon^{u}\right)^{1 / 3} \Omega_{0}^{-1} k_{\perp}^{5 / 3} .
$$


Using relations (28) and (30) we can derive the kinetic energy spectrum,

$$
E^{u}\left(k_{\perp}\right) \sim \frac{\widetilde{U}^{2}}{k_{\perp}} \sim\left(\epsilon^{u}\right)^{2 / 3} k_{\perp}^{-5 / 3} .
$$

The magnetic energy spectrum can be calculated similarly using the magnetostrophic equation in which the nonlinear time scale and the linear wave period are given by (19) and (20) respectively. Equating these two time scales gives a scaling for the wave amplitude as

$$
\widetilde{B} \sim \frac{k_{\|} b_{0}}{k_{\perp}}
$$

and the magnetic energy flux as

$$
\epsilon^{b} \sim \frac{\widetilde{B}^{2}}{\tau_{\text {tr }}}
$$

The CB scaling for the wavenumbers is thus

$$
k_{\|} \sim\left(\epsilon^{b}\right)^{1 / 3} b_{0}^{-1} d^{-1 / 3} k_{\perp}^{1 / 3},
$$

which leads to the magnetic energy spectrum

$$
E^{b}\left(k_{\perp}\right) \sim\left(\frac{\epsilon^{b}}{d}\right)^{2 / 3} k_{\perp}^{-7 / 3} .
$$

Expressions (34) and (35) are the new theoretical predictions for the strong wave turbulence of magnetostrophic waves which will be put to test (together with the previous predictions) by numerical simulations in the present paper.

\section{Numerical simulations}

\subsection{Set-up}

The rotating MHD equations (1)-(4) were solved numerically using the Fourier pseudospectral code GHOST [28-30]. Time integration is performed by a second-order Runge-Kutta scheme and the $2 / 3$ rule is employed for dealiasing. An isotropic initial condition of velocity and magnetic field fluctuations with random phases was chosen. We consider a decaying turbulence to avoid any artefacts from forcing. Note that, like in the classical Navier-Stokes turbulence, most of the energy in our system remains at or near the initial scales. This makes the results obtained theoretically for the force-dissipated system relevant to the unforced (decaying) turbulence. A hyperviscosity $\nu \nabla^{6} \boldsymbol{u}$ and hyperdiffusivity $\eta \nabla^{6} \boldsymbol{b}$ were used in place of the viscous and diffusive terms. As usual in turbulence simulations, using hyperviscosity and hyperdiffusivity allows to increase the effective inertial range. The degree and the coefficients are tuned so that there is no unphysical bottleneck bump at the highest wavenumbers.

In order to access the two regimes of rotating MHD turbulence, namely the WWT and the $\mathrm{CB}$ regimes, we will be interested in the large-scale limit,

$$
k d=k \frac{b_{0}}{\Omega_{0}} \ll 1,
$$

and the limit of small Rossby numbers, 
Table 1. Parameters used in the numerical simulations.

\begin{tabular}{lllllll}
\hline Simulation & $b_{0}$ & $\Omega_{0}$ & $\widetilde{U}$ & $\widetilde{B}$ & $\nu$ & $\eta$ \\
\hline$A$ & 50 & 1000 & 5.0 & 15.0 & $10^{-12}$ & $10^{-12}$ \\
$B$ & 50 & 1000 & 0.5 & 1.5 & $10^{-14}$ & $10^{-14}$ \\
\hline
\end{tabular}

$$
\text { Ro } \sim \frac{k U}{\Omega_{0}} \ll 1 .
$$

Again, these conditions may be well satisfied at one end of the wavenumber range (small $k^{\prime} s$ ) and only marginally at the other (large $k$ 's). The difference between the weak and strong turbulence regimes is controlled by the ratios of linear wave period to nonlinear turnover time given by equations (23) and (24). These ratios should be very small in the WTT limit and of the order 1 in the $\mathrm{CB}$.

\subsection{Simulation A: critical balance}

In simulation $A$ we have aimed to access the $\mathrm{CB}$ regime. The parameters used for the simulation can be found in table 1. A simple initial condition was chosen with all modes in the range $k=[2,4]$ having equal amplitudes and random phases. The simulation has been performed in a periodic box of spatial resolution $512^{3}$. After application of the $N / 3$ rule for dealiasing, this gives a maximum wavenumber of 170 . The parameter $k d$ therefore ranges from 0 to 8.5 . This is only moderately small for about a decade of the wavenumber range and thus complete decoupling of the magnetic and kinetic energies may not be achieved. However, it is sufficiently far from the opposite limit $k d \rightarrow \infty$ for this simulation, so the inertial and the magnetostrophic wave branches are well separated. The strength of the initial condition is such that the ratios of linear to nonlinear time scales are of the order unity as is expected for a $\mathrm{CB}$ state. We have also performed simulations in the $\mathrm{CB}$ regime at resolution $256^{3}$ for a qualitative study of robustness of the obtained spectra (more rigorous convergence study was out of our reach due to the smallness of the scaling range discussed above).

The $2 D$ energy spectra are plotted in figure 1 . For both the kinetic and magnetic energy there is a preferential transfer of energy along $k_{\perp}$ leading to the anisotropy $k_{\perp}>k_{\|}$. Figure 2 shows the axially averaged energy spectra integrated over $k_{\|}$. Each spectrum is compensated by the relevant $\mathrm{CB}$ prediction and is plotted alongside the WWT slope prediction for comparison. Both the kinetic and the magnetic energy spectra show scaling ranges in an excellent agreement with the CB predictions given by equations (31) and (35) respectively. The WWT prediction for the kinetic energy is clearly far from the observed spectrum. For the magnetic energy spectrum the WWT and the CB predictions are close, and they fit the observed spectrum equally well. However, the spatio-temporal spectrum presented below allow us to confidently conclude that what is observed here is a CB state and not WWT. To the left of the scaling ranges, at and near the initial scales, one can observe bumps on the spectrum. This behaviour is typical for the finite capacity systems where the evolution is much slower at the large scales than at the small ones, so that most of the energy remains at the largest scales by the time scalings develop at the small scales.

So far we have concentrated on the spatial properties of rotating MHD at a given point in time. In order to identify the presence of waves however, it is necessary to consider the properties in the spatio-temporal domain. A common method is to analyse the spatio-temporal spectrum [31-34]. This can then be compared with the dispersion relation of the linear waves. Computing the spatio-temporal spectrum requires simultaneous space and time Fourier 

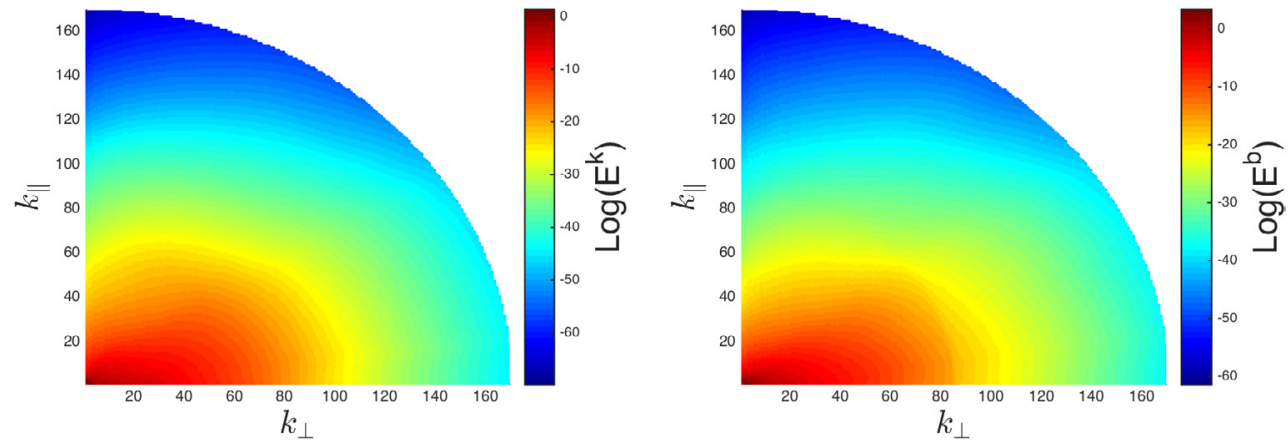

Figure 1. $2 D$ energy spectra for the (a) kinetic and (b) magnetic energies in simulation $A$.
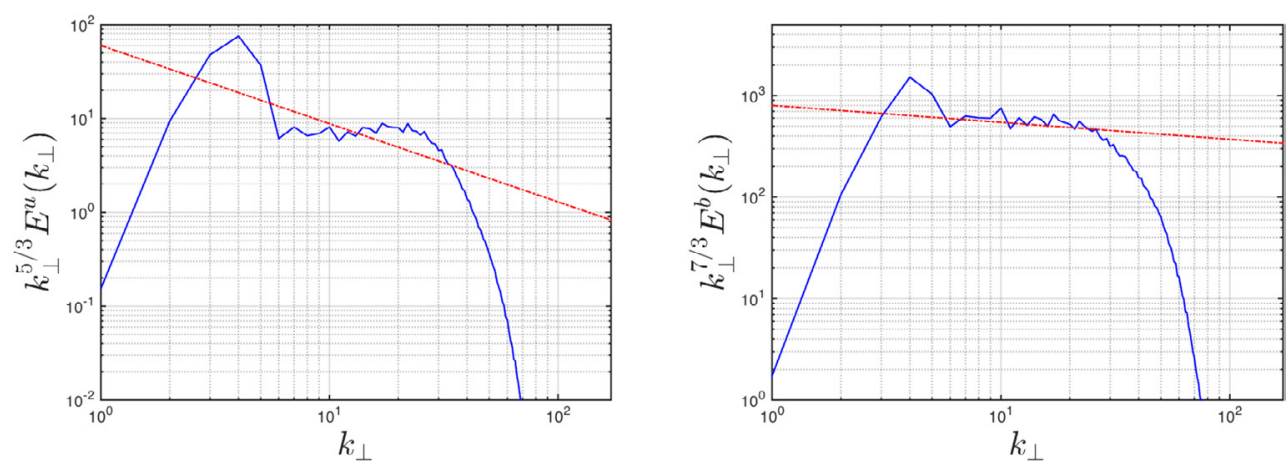

Figure 2. Polar angle averaged energy spectra for the (a) kinetic and (b) magnetic energies in simulation $A$ compensated by the CB spectra, equations (31) and (35) respectively. The straight lines indicate the WWT predictions for comparison, equations (18) and (21) respectively.

transforms. In order to resolve all the waves, the time sampling frequency must be at least twice as large as the frequency of the fastest waves in the system and the total acquisition time must be larger than both the slowest wave period and the turnover time of the slowest eddies. These requirements result in a high storage space requirement which proved restrictive, so a subset of data was collected for a fixed $k_{\|}=3$.

In figures 3 and 4 we plot the spatio-temporal spectra for the kinetic and magnetostrophic energy respectively. The spectra are calculated at a fixed $k_{\|}=3$ and overlayed with the inertial and magnetostrophic wave dispersion relations. In the WWT regime, one would expect the kinetic and magnetic energy to accumulate narrowly on and near the dispersion relations for the linear waves. In a CB regime however, the width of the distribution in the $\omega$-direction is of the same size as the linear wave frequency. It was noted before for the Alfven wave turbulence that in the $\mathrm{CB}$ regime the dispersion relation acts as a boundary with the energy filling the region below it [35]. In figure 4 we see that the magnetic energy behaves exactly like this with the energy filling the area in $\left(k_{\perp}, \omega\right)$ space between the two polarities of the magnetostrophic dispersion relation. In figure 3 we see that the kinetic energy behaves in a similar way at high wavenumbers but does not fill the region at small wavenumbers. The ratio of linear wave frequency to the inverse nonlinear turnover time becomes less than 1 at $k_{\perp}<24$ and we can clearly see a separation of two branches-weak waves and a low-frequency component, 


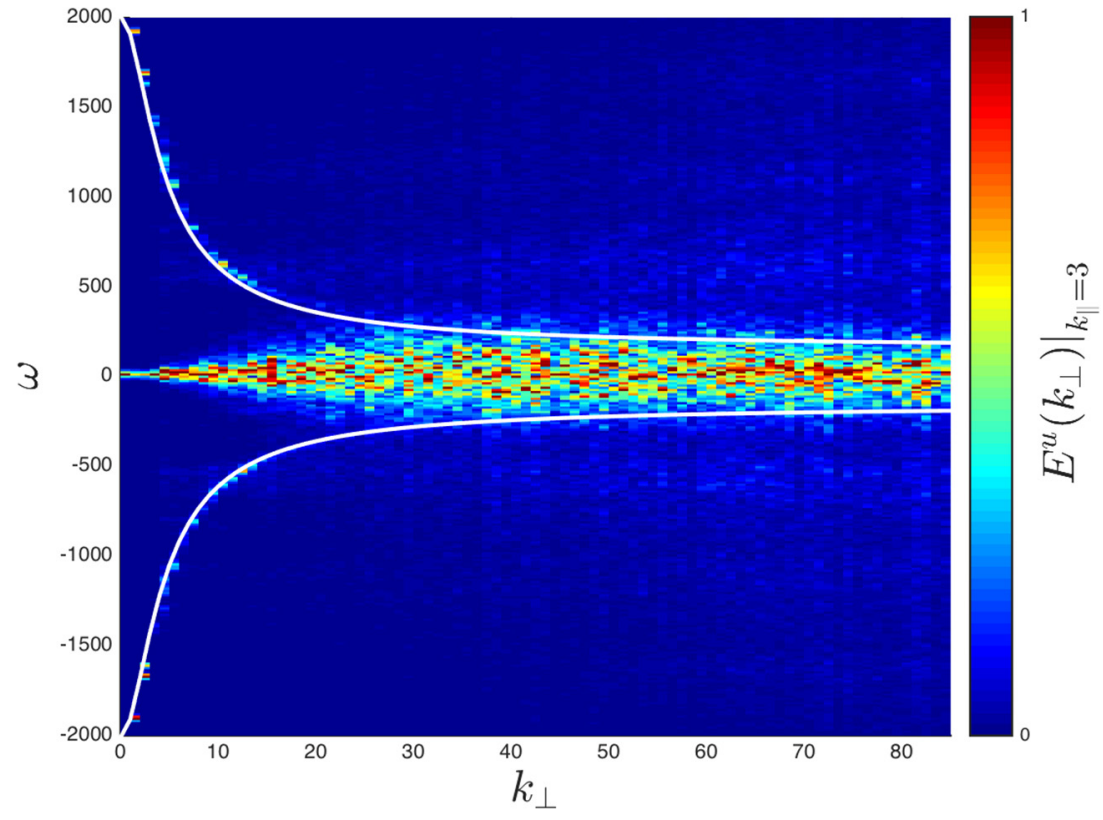

Figure 3. Spatio-temporal energy kinetic energy spectrum in simulation $A$. The white lines indicate the dispersion relation for inertial waves.

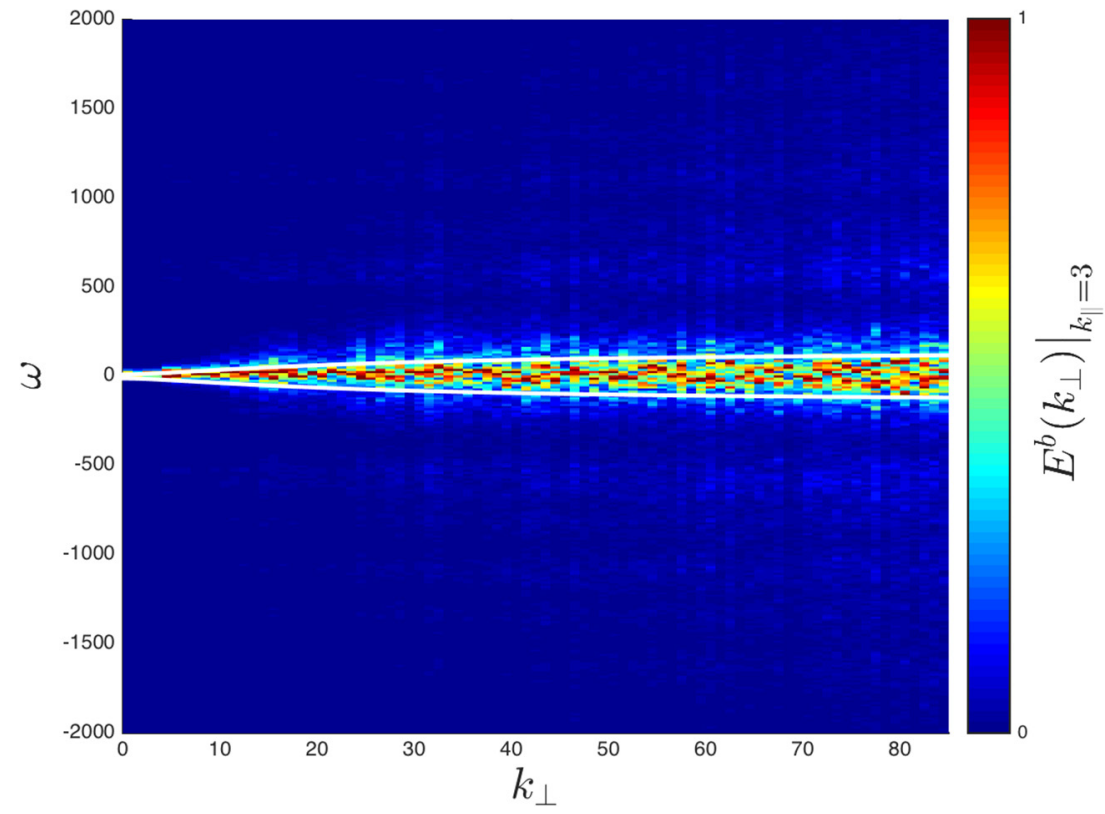

Figure 4. Spatio-temporal energy magnetic energy spectrum in simulation $A$. The white lines indicate the dispersion relation for magnetostrophic waves. 


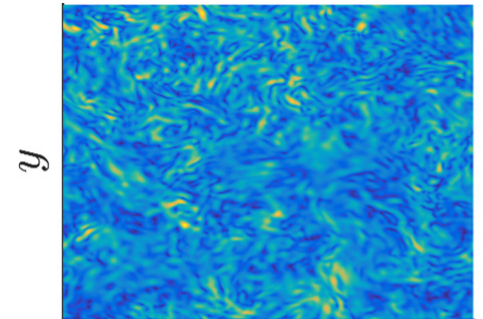

$x$

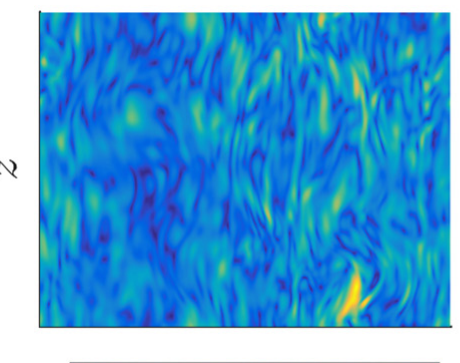

$20 \quad 4060 \quad 80$

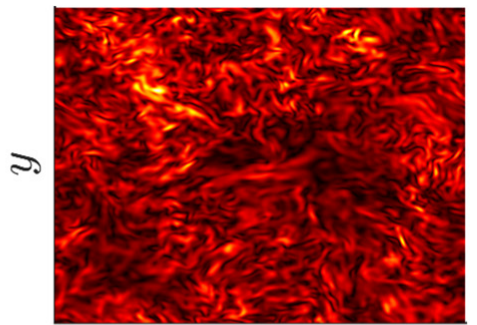

$x$

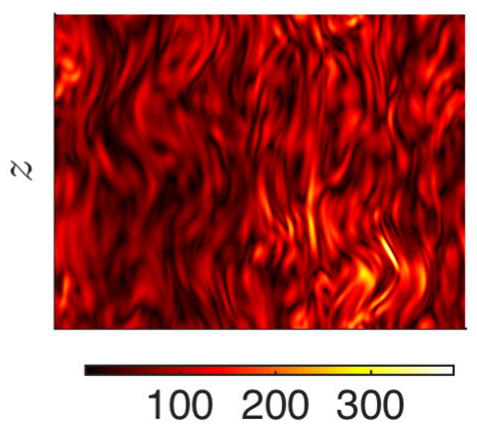

Figure 5. Simulation $A$ fields in the physical space. Left: vorticity amplitude $|\nabla \times \mathbf{u}|$ in the $\mathbf{x}_{\perp}=(x, y)$ plane and the $(x, z)$ plane. Right: current amplitude $|\nabla \times \mathbf{b}|$ in the $\mathbf{x}_{\perp}$ plane and the $(x, z)$ plane.
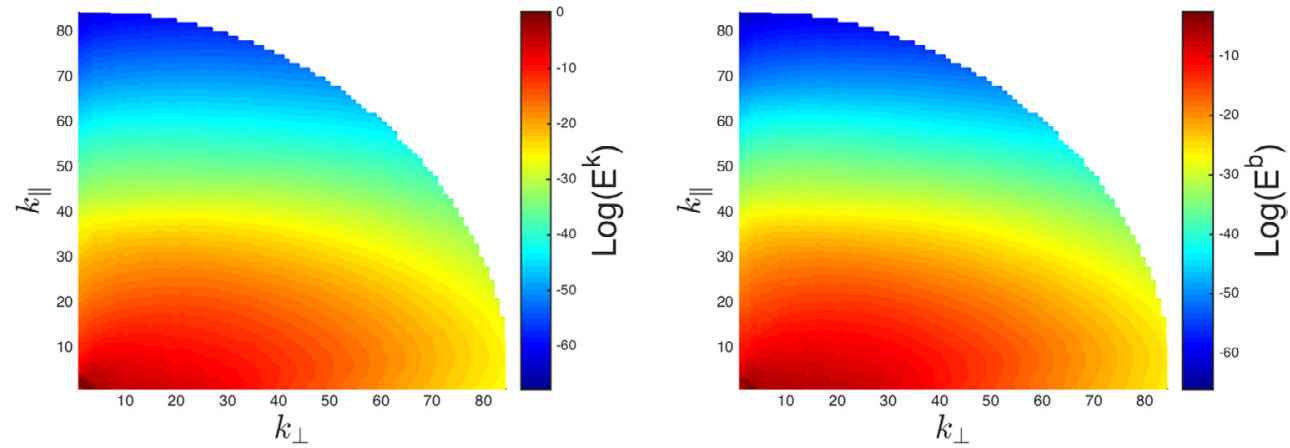

Figure 6. $2 D$ energy spectra for the (a) kinetic and (b) magnetic energies in simulation $B$.

presumably vortices. The CB concept is too simplified to describe such a two-component turbulence, and development of new approaches describing coexisting and interacting waves and vortices are needed. Interestingly, the CB prediction for the kinetic energy spectrum is observed for the wavenumbers both below and above $k_{\perp}=24$ without any particular feature observed at this transitional scale. One could argue that what is observed is just the classical Kolmogorov spectrum and not CB, but the evident strong anisotropy of the spectrum plays agains such a simple explanation.

In figure 5 we plot the amplitude of the vorticity and current both in the $\mathbf{x}_{\perp}=(x, y)$ plane and the $(x, z)$ plane. In the perpendicular plane, we see a collection of small-scale structures both in the current and in the vorticity which are isotropically and homogeneously distributed. 

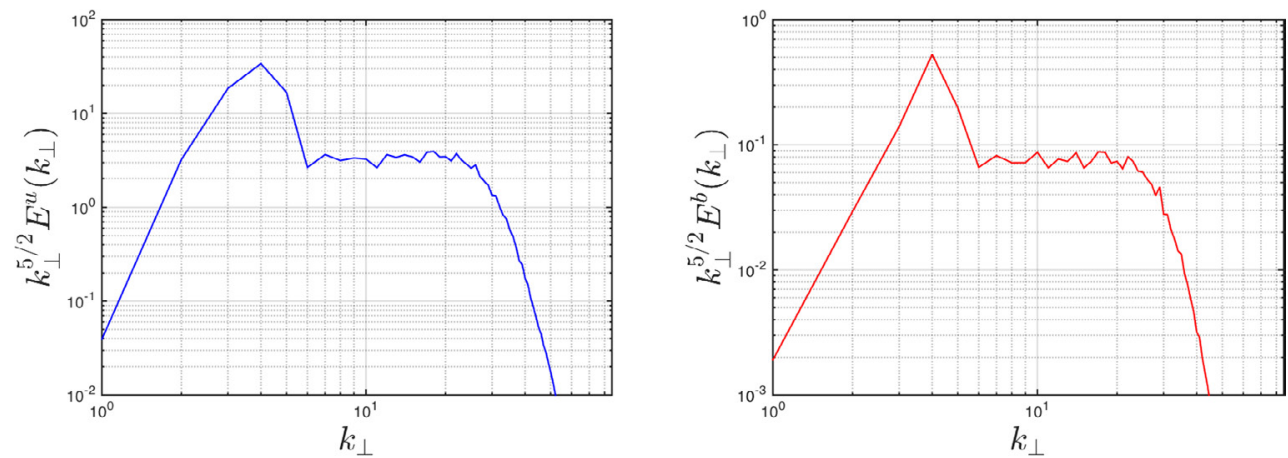

Figure 7. Polar angle averaged energy spectra for the (a) kinetic and (b) magnetic energies in simulation $B$ compensated by the WWT predictions, equations (18) and (21) respectively.

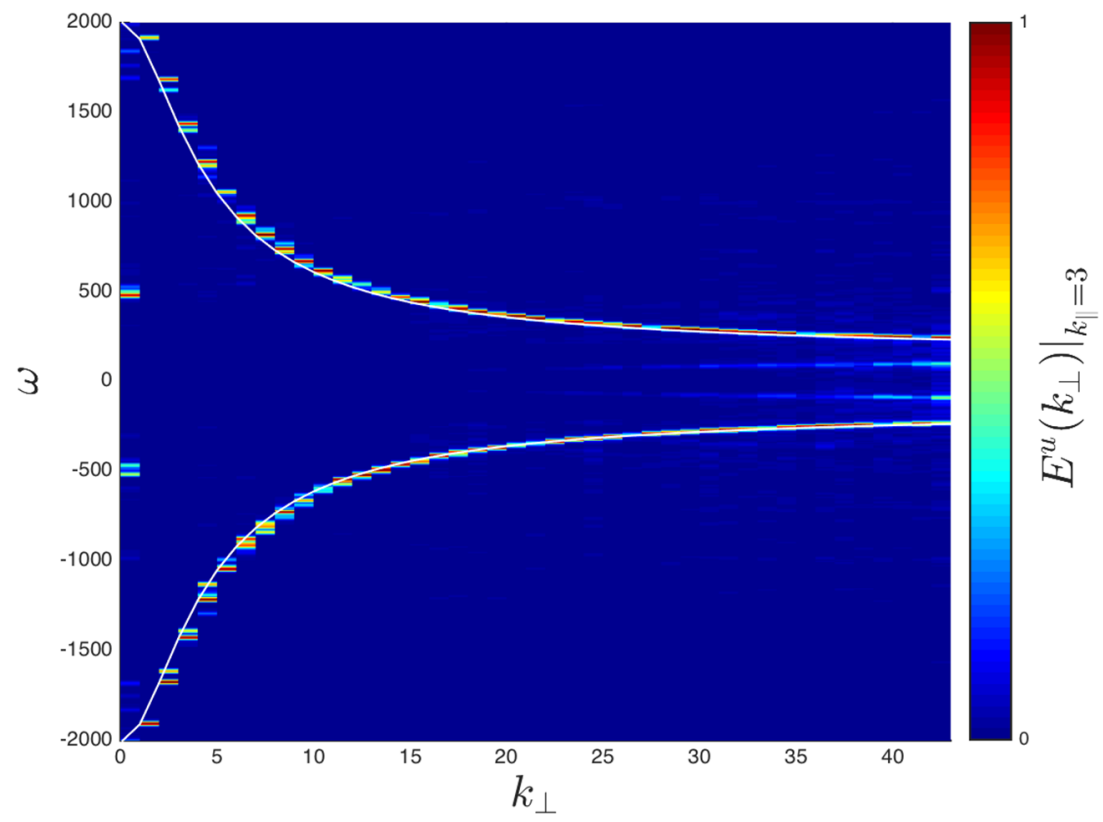

Figure 8. Spatio-temporal energy kinetic energy spectrum in simulation $B$. The white lines indicate the dispersion relation for inertial waves.

In the vertical plane see that the structures are stretched in the parallel direction as expected for an anisotropic state. Combined with the $2 D$ spectra in figure 1 , we have strong evidence for the anisotropy assumption which both the weak and strong turbulence predictions are based upon.

\subsection{Simulation B: weak wave turbulence}

In simulation $B$ we have reduced amplitude, and magnetic and kinetic energy (with respect to simulation $A$ ) in an attempt to access a regime relevant to WWT. The full set of parameters are again given in table 1 . With this parameter set, it was found that the development towards 


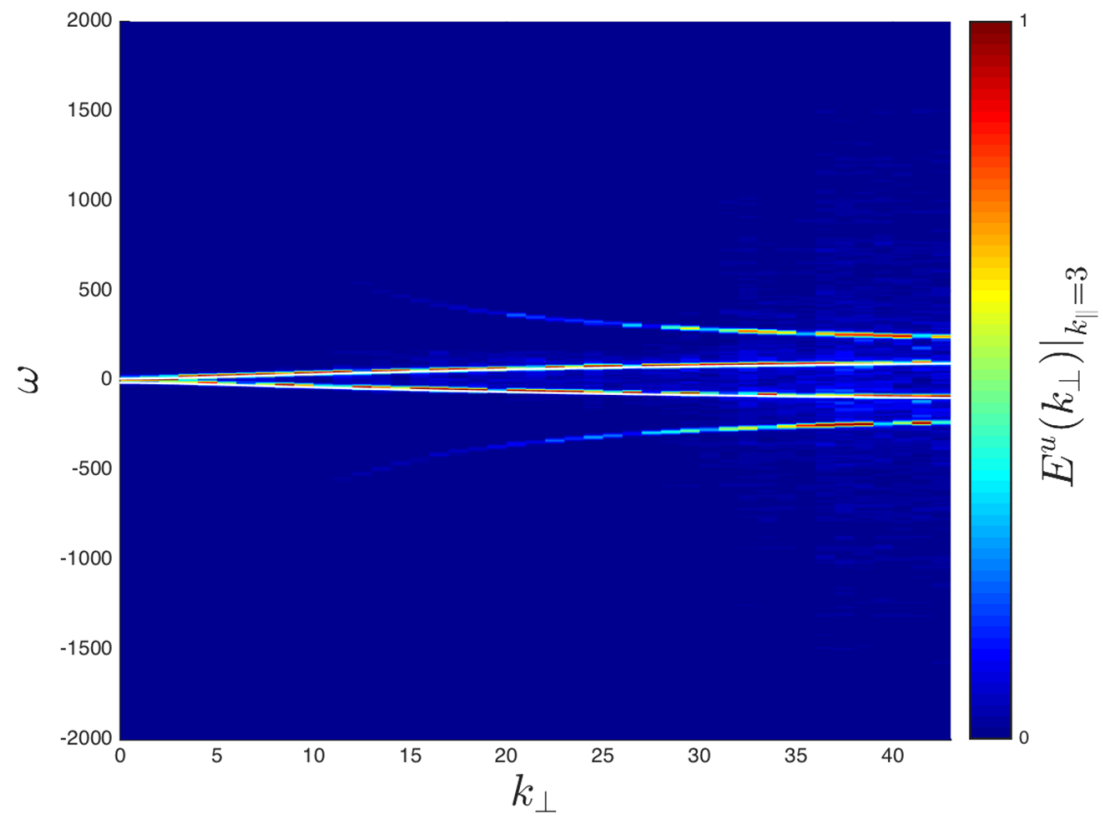

Figure 9. Spatio-temporal energy magnetic energy spectrum in simulation $B$. The white lines indicate the dispersion relation for magnetostrophic waves.

a stationary spectra was much slower than in simulation $A$. Thus, the simulation has been performed with a moderate resolution of $256^{3}$. Study of convergence of the spectra was further limited by the fact that at lower resolution (e.g. $128^{3}$ ) the wave resonances and the energy cascade are severely inhibited-this is the so-called discrete (or frozen) WT regime.

The $2 D$ energy spectra for simulation $B$ are plotted in figure 6 . As expected, for both the kinetic and magnetic energy there is a preferential transfer of energy along $k_{\perp}$. Figure 7 shows the polar angle averaged energy spectra integrated over $k_{\|}$. Each spectrum has been compensated by the WWT prediction, $k_{\perp}^{-5 / 2}$, and both appear to be flat, suggesting an agreement with the prediction.

As before, the presence of waves can be tested by plotting the spatio-temporal spectra for the magnetic and kinetic energy. This is done in figures 8 and 9. They are plotted alongside the dispersion relation for inertial and magnetostrophic waves respectively. In each case, we see a build up of a narrow energy distribution on and near the dispersion relation suggesting the presence of weak inertial and megnetostrophic waves. This combined with the apparent agreement between the energy spectra and the WWT prediction, leads us to believe that the dynamics that the WWT regime is realised in simulation $B$. Note that for $k_{\perp} \geqslant 25$ one can see two wave branches on each of the plots. This is reflection of the fact that at scales with $k d \geqslant 1$ both the inertial and the magnetostrophic modes contain comparable amounts of the kinetic and the magnetic energies. Our theoretical scaling predictions are not applicable for these scales.

The physical space distributions of the vorticity and the magnetic fields in the simulation $B$ appear to be similar (perhaps somewhat more anisotropic) to the ones in figure 5 for the simulation $A$; so we omit presenting separate images of these fields here. Chararterizing finer differences in morphology of the physical space structures in the weak and the strong turbulence cases is beyond the scope of our paper, although it could be an interesting subject for 
future research. This would allow, in particular, test CB relations between the parallel and the perpendicular scales (30) and (34).

\section{Conclusions}

In this paper we have studied turbulence in a rotating MHD system using direct numerical simulation of the governing equations. We have reviewed the weak wave turbulence in rotating MHD the theory of which was developed in [1]. We have also derived scalings for the kinetic and magnetic energy spectra for strong wave turbulence based upon the CB phenomenology which postulates the timescales of the linear waves and the eddies are of comparable magnitude.

Two simulations have been presented, one for a strong and a weak wave turbulence regimes respectively. As expected, and as previously observed for purely MHD and purely rotating turbulence cases, turbulence quickly evolves toward strongly anisotropic states with elongated along the external magnetic field vortex and current structures. In each case, we have presented the numerical results for the 2D (axial angle averaged) energy spectra, 1D energy spectra (obtained by integrating the $2 \mathrm{D}$ spectra over the parallel wavenumber) and spatiotemporal spectra at a fixed parallel wavenumber. For strong and weak wave turbulence, these results are in a good agreement with predictions of the CB and the WWT theoretical predictions, particularly in the part concerning the 1D spectra. The spatio-temporal plots have allowed us to differentiate between weak dispersive waves and strongly-turbulent structures, such as hydrodynamic vortices and, thereby, directly examine realisability of assumptions of weak nonlinearity and of CB. For instance, it allowed us the verify that the weak nonlinearity property in simulation $B$. However, for the strong nonlinearity run (simulation $A$ ) the spatiotemporal spectrum has revealed that the $\mathrm{CB}$ picture, where the frequency broadening is of the same size as the linear frequency, is somewhat oversimplified. Namely, in the low-wavenumber range the the spatio-temporal spectrum has a well-pronounced two-peaked distribution with a narrow peak at the linear frequency and a wide peak around the zero frequency. This indicates that a finer picture of strong wave turbulence in this case should contain two distinct dynamical components, weak waves and strong vortices (a 'condensate') which coexist and interact. Developing such a description is an interesting subject for future research.

\section{Acknowledgments}

Nicholas Bell was supported by EPSRC Grant 1499213 funding the PhD study. Sergey Nazarenko is supported by the Chaire D'Excellence IDEX (Initiative of Excellence) awarded by Université de la Côte d'Azur, France and by the Simons Foundation Collaboration grant 'Wave Turbulence' (Award ID 651471).

\section{Appendix}

The derivation of the WWT for rotating MHD was presented in [1], where the wave kinetic equations (WKEs) were derived. This was followed by finding the KZ spectra for the inertial and magnetostrophic waves under an assumption that these two types of waves are decoupled from each other. However, the decoupling property was simply assumed. In this appendix we will explore the conditions under which the decoupling assumption is valid. Further, in the second part we will find a regime where the nonlinear coupling between the inertial and magnitostrophic waves is important and will present simplified WKEs for such a regime. 
The full WKEs for for rotating MHD are as follows [1],

$$
\begin{aligned}
\frac{\partial n_{\Lambda}^{s}(\boldsymbol{k})}{\partial t}= & \frac{\pi \epsilon^{2} d^{4}}{64 b_{0}^{2}} \iint \sum_{\substack{\Lambda_{1}, \Lambda_{2} \\
s_{1}, s_{2}}}\left(\frac{\sin \psi_{k}}{k}\right)^{2} k^{2} k_{1}^{2} k_{2}^{2}\left(\Lambda k+\Lambda_{1} k_{1}+\Lambda_{2} k_{2}\right)^{2} \\
& \times\left(\xi_{\Lambda}^{s}\right)^{2}\left(\xi_{\Lambda_{1}}^{s_{1}}\right)^{2}\left(\xi_{\Lambda_{2}}^{s_{2}}\right)^{2}\left(\frac{\xi_{\Lambda_{2}}^{-s_{2}}-\xi_{\Lambda_{1}}^{-s_{1}}}{k_{\|}}\right)^{2}\left(\frac{\omega_{\Lambda}^{s}}{1+\left(\xi_{\Lambda}^{-s}\right)^{2}}\right) \\
& \times\left(2+\left(\xi_{\Lambda}^{-s}\right)^{2}\left(\xi_{\Lambda_{1}}^{-s_{1}}\right)^{2}\left(\xi_{\Lambda_{2}}^{-s_{2}}\right)^{2}-\left(\xi_{\Lambda}^{-s}\right)^{2}-\left(\xi_{\Lambda_{1}}^{-s_{1}}\right)^{2}-\left(\xi_{\Lambda_{2}}^{-s_{2}}\right)^{2}\right)^{2} \\
& \times\left[\frac{\omega_{\Lambda}^{s_{1}}}{\left\{1+\left(\xi_{\Lambda}^{-s}\right)^{2}\right\} n_{\Lambda}^{s}(\boldsymbol{k})}-\frac{\omega_{\Lambda_{2}}^{s_{2}}}{\left\{1+\left(\xi_{\Lambda_{1}}^{-s_{1}}\right)^{2}\right\} n_{\Lambda_{1}}^{s_{1}}\left(\boldsymbol{k}_{\mathbf{1}}\right)}-\frac{\left.\omega_{1}^{s}+\left(\xi_{\Lambda_{2}}^{-s_{2}}\right)^{2}\right\} n_{\Lambda_{2}}^{s_{2}}\left(\boldsymbol{k}_{\mathbf{2}}\right)}{\{1}\right] \\
& \times n_{\Lambda}^{s}(\boldsymbol{k}) n_{\Lambda_{1}}^{s_{1}}\left(\boldsymbol{k}_{\mathbf{1}}\right) n_{\Lambda_{2}}^{s_{2}}\left(\boldsymbol{k}_{\mathbf{2}}\right) \delta\left(\omega_{\Lambda_{1}}^{s_{1}}+\omega_{\Lambda_{2}}^{s_{2}}-\omega_{\Lambda}^{s}\right) \delta\left(\boldsymbol{k}-\boldsymbol{k}_{\mathbf{1}}-\boldsymbol{k}_{\mathbf{2}}\right) \mathrm{d} \boldsymbol{k}_{\mathbf{1}} \mathrm{d} \boldsymbol{k}_{\mathbf{2}} .
\end{aligned}
$$

The angle $\psi_{k}$ refers to the angle opposite $\boldsymbol{k}$ in the triangle defined by $\boldsymbol{k}=\boldsymbol{k}_{\mathbf{1}}+\boldsymbol{k}_{\mathbf{2}}$, $\xi_{\Lambda}^{s}$ is defined by

$$
\xi_{\Lambda}^{s}=\frac{-s k d}{-s \Lambda+\sqrt{1+k^{2} d^{2}}} .
$$

Here $s= \pm$ defines the directional wave polarity and $\Lambda= \pm s$ defines the circular polarization. If $\Lambda=s$ then we are dealing with the right polarized wave which is the magnetostrophic wave in this case. The left polarized waves are given by $\Lambda=-s$ and correspond to inertial waves. Solving the full kinetic equation would be extremely difficult even numerically. Thus, one tends to reduce the WKEs by considering by considering relevant special cases.

First we shall consider the case when parameter $k d$ is small. Consider first the equation describing the dynamics of the inertial waves $(\Lambda=-s)$. Performing the summation over polarisations $\Lambda_{1}$ and $\Lambda_{2}$, the kinetic equation takes the form

$$
\partial_{t} n_{-s}^{s}(\boldsymbol{k})=A_{\mathrm{II}}+B_{\mathrm{IM}}+C_{\mathrm{MM}},
$$

where $A_{\text {II }}$ gives the contribution from inertial-inertial wave interactions, $B_{\mathrm{IM}}$ gives the contribution from inertial-magnetostrophic wave interactions and $C_{\mathrm{MM}}$ gives the contribution from magnetostrophic-magnetostrophic interactions. Now, to leading order in $k d$ we have the following expansions, $\xi_{-s}^{s} \rightarrow-\frac{s k d}{2}, \xi_{s}^{s} \rightarrow-\frac{2 s}{k d}, \omega_{s}^{s} \rightarrow \frac{s k_{\|} k \mathrm{~d} b_{0}}{2}=\omega_{\mathrm{M}}, \omega_{-s}^{s} \rightarrow \frac{2 s \Omega_{0} k_{\|}}{k}=\omega_{\mathrm{I}}$. Using these expansions, we write the asymptotic expressions for the terms in equation (A.3) as

$$
\begin{gathered}
A_{\mathrm{II}}=\frac{\pi \epsilon^{2}}{4 b_{0}^{2}} \int \sum_{s_{1}, s_{2}}\left(\frac{\sin \psi_{k}}{k}\right)^{2}\left(s k+s_{1} k_{1}+s_{2} k_{2}\right)^{2} \frac{\left(s_{2} k_{1}-s_{1} k_{2}\right)^{2}}{k_{1}^{2} k_{2}^{2}} \\
\times \frac{k^{2} \omega_{-s}^{s}}{k_{\|}^{2}} n_{-s}^{s} n_{-s_{1}}^{s_{1}} n_{-s_{2}}^{s_{2}}\left[\frac{k^{2} \omega_{-s}^{s}}{n_{-s}^{s}}-\frac{k_{1}^{2} \omega_{-s_{1}}^{s_{1}}}{n_{-s_{1}}^{s_{1}}}-\frac{k_{2}^{2} \omega_{-s_{2}}^{s_{2}}}{n_{-s_{2}}^{s_{2}}}\right] \\
\times \delta\left(\omega_{-s_{1}}^{s_{1}}+\omega_{-s_{2}}^{s_{2}}-\omega_{-s}^{s}\right) \delta\left(\boldsymbol{k}-\boldsymbol{k}_{\mathbf{1}}-\boldsymbol{k}_{\mathbf{2}}\right) \mathrm{d} \boldsymbol{k}_{1} \mathrm{~d} \boldsymbol{k}_{2}, \\
B_{\mathrm{IM}}=\frac{\pi \epsilon^{2}}{8 b_{0}^{2}} \int \sum_{s_{1}, s_{1}} k^{2} d^{2}\left(\frac{\sin \psi_{k}}{k}\right)^{2}\left(s k+s_{1} k_{1}+s_{2} k_{2}\right)^{2} \frac{\left(k_{2}^{2}-k_{1}^{2}-k^{2}\right)^{2}}{k_{1}^{2}} \frac{\omega_{-s}^{s}}{k_{\|}^{2}} n_{-s}^{s} n_{-s_{1}}^{s_{1}} n_{s_{2}}^{s_{2}} \\
\times\left[\frac{k^{2} d^{2} \omega_{-s}^{s}}{4 n_{-s}^{s}}-\frac{k_{1}^{2} d^{2} \omega_{-s_{1}}^{s_{1}}}{4 n_{-s_{1}}^{s_{1}}}-\frac{\omega_{s_{2}}^{s_{2}}}{n_{s_{2}}^{s_{2}}}\right] \delta\left(\omega_{-s_{1}}^{s_{1}}+\omega_{s_{2}}^{s_{2}}-\omega_{-s}^{s}\right) \delta\left(\boldsymbol{k}-\boldsymbol{k}_{\mathbf{1}}-\boldsymbol{k}_{\mathbf{2}}\right) \mathrm{d} \boldsymbol{k}_{1} \mathrm{~d} \boldsymbol{k}_{2},
\end{gathered}
$$




$$
\begin{aligned}
C_{\mathrm{MM}} & =\frac{\pi \epsilon^{2}}{16 b_{0}^{2}} \int \sum_{s_{1}, s_{2}} k^{2} d^{2}\left(\frac{\sin \psi_{k}}{k}\right)^{2}\left(s k+s_{1} k_{1}+s_{2} k_{2}\right)^{2}\left(s_{2} k_{2}-s_{1} k_{1}\right)^{2} \frac{\omega_{-s}^{s}}{k_{\|}^{2}} n_{-s}^{s} n_{s_{1}}^{s_{1}} n_{s_{2}}^{s_{2}} \\
& \times\left[\frac{k^{2} d^{2} \omega_{-s}^{s}}{4 n_{-s}^{s}}-\frac{\omega_{s_{1}}^{s_{1}}}{n_{s_{1}}^{s_{1}}}-\frac{\omega_{s_{2}}^{s_{2}}}{n_{s_{2}}^{s_{2}}}\right] \delta\left(\omega_{s_{1}}^{s_{1}}+\omega_{s_{2}}^{s_{2}}-\omega_{-s}^{s}\right) \delta\left(\boldsymbol{k}-\boldsymbol{k}_{\mathbf{1}}-\boldsymbol{k}_{\mathbf{2}}\right) \mathrm{d} \boldsymbol{k}_{1} \mathrm{~d} \boldsymbol{k}_{2} .
\end{aligned}
$$

Now consider the equation describing the dynamics of the magnetostrophic wave action, $n_{s}^{s}$. The WKE will assume a similar form as for inertial waves,

$$
\partial_{t} n_{s}^{s}(\boldsymbol{k})=D_{\mathrm{MM}}+E_{\mathrm{IM}}+F_{\mathrm{II}} .
$$

The above expansions are again used to give the individual terms as

$$
\begin{aligned}
& D_{\mathrm{MM}}=\frac{\pi \epsilon^{2}}{b_{0}^{2}} \int \sum_{s_{1}, s_{2}}\left(\frac{\sin \psi_{k}}{k}\right)^{2}\left(s k+s_{1} k_{1}+s_{2} k_{2}\right)^{2}\left(s_{1} k_{1}-s_{2} k_{2}\right)^{2} \frac{\omega_{s}^{s}}{k_{\|}^{2}} \\
& \times n_{s}^{s} n_{s_{1}}^{s_{1}} n_{s_{2}}^{s_{2}}\left[\frac{\omega_{s}^{s}}{n_{s}^{s}}-\frac{\omega_{s_{1}}^{s_{1}}}{n_{s_{1}}^{s_{1}}}-\frac{\omega_{s_{2}}^{s_{2}}}{n_{s_{2}}^{s_{2}}}\right] \delta\left(\omega_{s_{1}}^{s_{1}}+\omega_{s_{2}}^{s_{2}}-\omega_{s}^{s}\right) \delta\left(\boldsymbol{k}-\boldsymbol{k}_{\mathbf{1}}-\boldsymbol{k}_{\mathbf{2}}\right) \mathrm{d} \boldsymbol{k}_{1} \mathrm{~d} \boldsymbol{k}_{2}, \\
& E_{\mathrm{IM}}=\frac{8 \pi \epsilon^{2}}{b_{0}^{2}} \int \sum_{s_{1}, s_{2}}\left(\frac{\sin \psi_{k}}{k}\right)^{2}\left(s k+s_{1} k_{1}+s_{2} k_{2}\right)^{2} \frac{\omega_{s}^{s}}{k_{\|}^{2} k_{1}^{2} d^{4}} n_{s}^{s} n_{-s_{1}}^{s_{1}} n_{s_{2}}^{s_{2}} \\
& \times\left[\frac{\omega_{s}^{s}}{n_{s}^{s}}-\frac{k_{1}^{2} d^{2} \omega_{-s_{1}}^{s_{1}}}{4 n_{-s_{1}}^{s_{1}}}-\frac{\omega_{s_{2}}^{s_{2}}}{n_{s_{2}}^{s_{2}}}\right] \delta\left(\omega_{-s_{1}}^{s_{1}}+\omega_{s_{2}}^{s_{2}}-\omega_{s}^{s}\right) \delta\left(\boldsymbol{k}-\boldsymbol{k}_{\mathbf{1}}-\boldsymbol{k}_{\mathbf{2}}\right) \mathrm{d} \boldsymbol{k}_{1} \mathrm{~d} \boldsymbol{k}_{2}, \\
& F_{\mathrm{II}}=\frac{\pi \epsilon^{2}}{4 b_{0}^{2}} \int \sum_{s_{1}, s_{2}}\left(\frac{\sin \psi_{k}}{k}\right)^{2}\left(s k+s_{1} k_{1}+s_{2} k_{2}\right)^{2}\left(s_{2} k_{1}-s_{1} k_{2}\right)^{2} \\
& \times \frac{\left(k^{2}-k_{1}^{2}-k_{2}^{2}\right)^{2}}{k_{1}^{2} k_{2}^{2}} \frac{\omega_{s}^{s}}{k_{\|}^{2}} n_{s}^{s} n_{-s_{1}}^{s_{1}} n_{-s_{2}}^{s_{2}}\left[\frac{\omega_{s}^{s}}{n_{s}^{s}}-\frac{k_{1}^{2} d^{2} \omega_{-s_{1}}^{s_{1}}}{4 n_{-s_{1}}^{s_{1}}}-\frac{k_{2}^{2} d^{2} \omega_{-s_{2}}^{s_{2}}}{4 n_{-s_{2}}^{s_{2}}}\right] \\
& \times \delta\left(\omega_{-s_{1}}^{s_{1}}+\omega_{-s_{2}}^{s_{2}}-\omega_{s}^{s}\right) \delta\left(\boldsymbol{k}-\boldsymbol{k}_{\mathbf{1}}-\boldsymbol{k}_{\mathbf{2}}\right) \mathrm{d} \boldsymbol{k}_{1} \mathrm{~d} \boldsymbol{k}_{2} \text {. }
\end{aligned}
$$

\section{A.1. Decoupled kinetic equations}

KZ spectra were derived in [1] assuming decoupling of the WKEs for the inertial and the magnetostrophic waves. Here, we will look at the decoupling property more closely. Let us assume that the dynamically important wavenumbers are of the same order for both the inertial waves and the magnetostrophic waves,

$$
k \sim k_{\|} \sim 1
$$

the anisotropic assumption is made later. In terms of our small parameter $k d \equiv \lambda$, we get the following scalings, $b_{0} \sim \lambda, \Omega_{0} \sim 1, \omega_{s}^{s} \sim \lambda^{2}, \omega_{-s}^{s} \sim 1, n_{s}^{s} \sim 1 / \lambda^{2}, n_{-s}^{s} \sim 1$. The scaling for the wave actions $n_{s}^{s}$ and $n_{-s}^{s}$ come from ensuring that the total energy contained within the inertial waves is of the same order as the total energy in the magnetostrophic waves,

$$
\int \omega_{-s}^{s} E_{-s}^{s}(\boldsymbol{k}) \mathrm{d} \boldsymbol{k} \sim \int \omega_{s}^{s} E_{s}^{s}(\boldsymbol{k}) \mathrm{d} \boldsymbol{k},
$$


together with the following relation for the energy given by [1]

$$
E_{\Lambda}^{s}(\boldsymbol{k})=\left[1+\left(\xi_{\Lambda}^{-s}\right)^{2}\right] n_{\Lambda}^{s}(\boldsymbol{k}) .
$$

Now one can use the above scalings to compare the relative magnitudes of the terms in equation (A.3). One can immediately neglect $C_{\mathrm{MM}}$ because $\delta\left(\omega_{-s}^{s}-\omega_{s_{1}}^{s_{1}}-\omega_{s_{2}}^{s_{2}}\right)=0$ if condition (A.11) is satisfied (since in this case $\omega_{-s}^{s} \gg \omega_{s}^{s}$ ). The remaining two terms scale as $A_{\mathrm{II}} \sim 1 / \lambda^{2}, B_{\mathrm{IM}} \sim 1$, and thus $B_{\mathrm{IM}}$ is negligible and we get the following WKE,

$$
\begin{aligned}
\partial_{t} n_{-s}^{s}(\boldsymbol{k})=\frac{\pi \epsilon^{2}}{4 b_{0}^{2}} \int \sum_{s_{1}, s_{2}}\left(\frac{\sin \psi_{k}}{k}\right)^{2}\left(s k+s_{1} k_{1}+s_{2} k_{2}\right)^{2} \frac{\left(s_{2} k_{1}-s_{1} k_{2}\right)^{2}}{k_{1}^{2} k_{2}^{2}} \\
\quad \times \frac{k^{2} \omega_{-s}^{s}}{k_{\|}^{2}} n_{-s}^{s} n_{-s_{1}}^{s_{1}} n_{-s_{2}}^{s_{2}}\left[\frac{k^{2} \omega_{-s}^{s}}{n_{-s}^{s}}-\frac{k_{1}^{2} \omega_{-s_{1}}^{s_{1}}}{n_{-s_{1}}^{s_{1}}}-\frac{k_{2}^{2} \omega_{-s_{2}}^{s_{2}}}{n_{-s_{2}}^{s_{2}}}\right] \\
\times \delta\left(\omega_{-s_{1}}^{s_{1}}+\omega_{-s_{2}}^{s_{2}}-\omega_{s}^{s}\right) \delta\left(\boldsymbol{k}-\boldsymbol{k}_{\mathbf{1}}-\boldsymbol{k}_{\mathbf{2}}\right) \mathrm{d} \boldsymbol{k}_{1} \mathrm{~d} \boldsymbol{k}_{2} .
\end{aligned}
$$

Same reasoning can now be applied to equation (A.7) describing the magnetostrophic waves. Therm $E_{\mathrm{IM}}$ is zero, since $\delta\left(\omega_{s}^{s}-\omega_{-s_{1}}^{s_{1}}-\omega_{s_{2}}^{s_{2}}\right)=0$, and $D_{\mathrm{MM}} \sim 1 / \lambda^{2}, F_{\mathrm{II}} \sim 1$. Thus, the term containing only magnetostrophic waves is dominant and the WKE becomes

$$
\begin{aligned}
\partial_{t} n_{s}^{s}(\boldsymbol{k})= & \frac{\pi \epsilon^{2}}{b_{0}^{2}} \int \sum_{s_{1}, s_{2}}\left(\frac{\sin \psi_{k}}{k}\right)^{2}\left(s k+s_{1} k_{1}+s_{2} k_{2}\right)^{2}\left(s_{1} k_{1}-s_{2} k_{2}\right)^{2} \frac{\omega_{s}^{s}}{k_{\|}^{2}} \\
& \times n_{s}^{s} n_{s_{1}}^{s_{1}} n_{s_{2}}^{s_{2}}\left[\frac{\omega_{s}^{s}}{n_{s}^{s}}-\frac{\omega_{s_{1}}^{s_{1}}}{n_{s_{1}}^{s_{1}}}-\frac{\omega_{s_{2}}^{s_{2}}}{n_{s_{2}}^{s_{2}}}\right] \delta\left(\omega_{s_{1}}^{s_{1}}+\omega_{s_{2}}^{s_{2}}-\omega_{s}^{s}\right) \delta\left(\boldsymbol{k}-\boldsymbol{k}_{\mathbf{1}}-\boldsymbol{k}_{\mathbf{2}}\right) \mathrm{d} \boldsymbol{k}_{1} \mathrm{~d} \boldsymbol{k}_{2} .
\end{aligned}
$$

Thus, the WKEs for the inertial and magnetostrophic waves are completely decoupled in the limit $k d \rightarrow 0$ if the wavenumbers of the both types of waves are of similar magnitudes and the energy densities are comparable.

\section{A.2. Coupled kinetic equations}

A regime in which this decoupling did not occur such that there was a transfer of energy between the two types of waves could be interesting dynamically. This occurs when terms $C_{\mathrm{MM}}$ and $E_{\mathrm{IM}}$ are non-zero i.e. if $\omega_{\mathrm{I}}=\omega_{-s}^{s}=\frac{2 \Omega_{0} s k_{\|}}{k}$ and $\omega_{\mathrm{M}}=\omega_{s}^{s}=\frac{s k_{\|} k \mathrm{~d} b_{0}}{2}$ are of the same order of magnitude. This can be achieved if we consider the wavenumbers for the inertial waves such that $k=\mathcal{O}(1)$ and $k_{\|}=\mathcal{O}\left(\lambda^{2}\right)$. whereas for the magnetostrophic wavenumbers we still have $k \sim k_{\|}=\mathcal{O}(1)$. The frequencies and the wave action spectra now scale as follows, $\omega_{-s}^{s} \sim \lambda^{2}, \omega_{s}^{s} \sim \lambda^{2}, n_{-s}^{s} \sim 1, n_{s}^{s} \sim 1$, where once again the energy in the inertial and magnetostrophic waves is assumed to be of the same order of magnitude.

Now that the frequencies are all of the same order, no terms are zero due to the frequency resonance condition. However, $B_{\mathrm{IM}}$ and $F_{\mathrm{II}}$ are zero due to the delta-function $\delta\left(\boldsymbol{k}-\boldsymbol{k}_{\mathbf{1}}-\boldsymbol{k}_{\mathbf{2}}\right)=\delta\left(\boldsymbol{k}_{\perp}-\boldsymbol{k}_{1 \perp}-\boldsymbol{k}_{2 \perp}\right) \delta\left(k_{\|}-k_{1 \|}-k_{2 \|}\right)$. The parallel wavenumber delta-function in $B_{\mathrm{IM}}$ is zero because $k_{2 \|}$ is large whereas $k_{\|}$and $k_{1 \|}$ are small. The $F_{\mathrm{II}}$ term is zero due to the same argument. The remaining terms scale as $A_{\mathrm{II}} \sim 1 / \lambda^{2}, C_{\mathrm{MM}} \sim 1 / \lambda^{2}, D_{\mathrm{MM}} \sim 1, E_{\mathrm{IM}} \sim 1 / \lambda^{2}$. From this we have to leading order, 


$$
\begin{aligned}
\partial_{t} n_{-s}^{s}(\boldsymbol{k})= & \frac{\pi \epsilon^{2}}{4 b_{0}^{2}} \int \sum_{s_{1}, s_{2}}\left(\frac{\sin \psi_{k}}{k}\right)^{2}\left(s k+s_{1} k_{1}+s_{2} k_{2}\right)^{2} \frac{\left(s_{2} k_{1}-s_{1} k_{2}\right)^{2}}{k_{1}^{2} k_{2}^{2}} \\
& \times \frac{k^{2} \omega_{-s}^{s}}{k_{\|}^{2}} n_{-s}^{s} n_{-s_{1}}^{s_{1}} n_{-s_{2}}^{s_{2}}\left[\frac{k^{2} \omega_{-s}^{s}}{n_{-s}^{s}}-\frac{k_{1}^{2} \omega_{-s_{1}}^{s_{1}}}{n_{-s_{1}}^{s_{1}}}-\frac{k_{2}^{2} \omega_{-s_{2}}^{s_{2}}}{n_{-s_{2}}^{s_{2}}}\right] \\
& \times \delta\left(\omega_{-s_{1}}^{s_{1}}+\omega_{-s_{2}}^{s_{2}}-\omega_{-s}^{s}\right) \delta\left(\boldsymbol{k}-\boldsymbol{k}_{\mathbf{1}}-\boldsymbol{k}_{\mathbf{2}}\right) \mathrm{d} \boldsymbol{k}_{1} \mathrm{~d} \boldsymbol{k}_{2} \\
- & \frac{\pi \epsilon^{2}}{16 b_{0}^{2}} \int \sum_{s_{1}, s_{2}} k^{2} d^{2}\left(\frac{\sin \psi_{k}}{k}\right)^{2}\left(s k+s_{1} k_{1}+s_{2} k_{2}\right)^{2}\left(s_{2} k_{2}-s_{1} k_{1}\right)^{2} \frac{\omega_{-s}^{s}}{k_{\|}^{2}} \\
\times & n_{-s}^{s} n_{s_{1}}^{s_{1}} n_{s_{2}}^{s_{2}}\left[\frac{\omega_{s_{1}}^{s_{1}}}{n_{s_{1}}^{s_{1}}}+\frac{\omega_{s_{2}}^{s_{2}}}{n_{s_{2}}^{s_{2}}}\right] \delta\left(\omega_{s_{1}}^{s_{1}}+\omega_{s_{2}}^{s_{2}}-\omega_{-s}^{s}\right) \delta\left(\boldsymbol{k}-\boldsymbol{k}_{\mathbf{1}}-\boldsymbol{k}_{\mathbf{2}}\right) \mathrm{d} \boldsymbol{k}_{1} \mathrm{~d} \boldsymbol{k}_{2} .
\end{aligned}
$$

Similarly, the magnetostrophic WKE is

$$
\begin{aligned}
\partial_{t} n_{s}^{s}(\boldsymbol{k}) & =\frac{8 \pi \epsilon^{2} \Omega^{4}}{b_{0}^{6}} \int \sum_{s_{1}, s_{2}}\left(\frac{\sin \psi_{k}}{k}\right)^{2}\left(s k+s_{1} k_{1}+s_{2} k_{2}\right)^{2} \frac{\omega_{s}^{s}}{k_{\|}^{2} k_{1}^{2}} \\
& \times n_{s}^{s} n_{-s_{1}}^{s_{1}} n_{s_{2}}^{s_{2}}\left[\frac{\omega_{s}^{s}}{n_{s}^{s}}-\frac{\omega_{s_{2}}^{s_{2}}}{n_{s_{2}}^{s_{2}}}\right] \delta\left(\omega_{-s_{1}}^{s_{1}}+\omega_{s_{2}}^{s_{2}}-\omega_{s}^{s}\right) \delta\left(\boldsymbol{k}-\boldsymbol{k}_{\mathbf{1}}-\boldsymbol{k}_{\mathbf{2}}\right) \mathrm{d} \boldsymbol{k}_{1} \mathrm{~d} \boldsymbol{k}_{2} .
\end{aligned}
$$

Equations (A.16) and (A.17) describe the kinetics of the inertial and magnetostrophic wave action spectra in a regime where there is coupling between the two types of wave. This regime is realised when there is a scale separation between the waves such that the perpendicular wavenumber dominates the parallel wavenumber in inertial range but are of the same scale in magnetostrophic waves. This setup points at an interesting dynamical regime where there may be significant transfer between kinetic and magnetic energy.

\section{ORCID iDs}

N K Bell @ https://orcid.org/0000-0002-6420-7735

S V Nazarenko (i) https://orcid.org/0000-0002-8614-4907

\section{References}

[1] Galtier S 2014 J. Fluid Mech. 757 114-54

[2] Galtier S, Nazarenko S, Newell A C and Pouquet A 2000 J. Plasma Phys. 63 447-88

[3] Galtier S and Bhattacharjee A 2005 Plasma Phys. Control Fusion 47 B791

[4] Galtier S 2006 J. Plasma Phys. 72 721-69

[5] Nazarenko S 2007 New J. Phys. 9307

[6] Schekochihin A A, Nazarenko S V and Yousef T A 2012 Phys. Rev. E 85036406

[7] Zakharov V E, L'vov V S and Falkovich G 1992 Kolmogorov Spectra of Turbulence 1: Wave Turbulence (Berlin: Springer)

[8] Nazarenko S 2011 Wave Turbulence (Lecture Notes in Physics) (Berlin: Springer)

[9] Goldreich P and Sridhar S 1995 Astrophys. J. 438 763-75

[10] Finlay C C and Jackson A 2003 Science 300 2084-6

[11] Finlay C C 2008 Waves in the presence of magnetic fields, rotation and convection Dynamos vol 88 ed P Cardin (Amsterdam: Elsevier) pp 403-50

[12] Romanova M M, Ustyugova G V, Koldoba A V and Lovelace R V E 2013 Mon. Not. R. Astron. Soc. 430 699-724

[13] Menu M D, Galtier S and Petitdemange L 2019 Phys. Rev. Fluids 4073701 
[14] Shirley J H and Fairbridge R W 1997 Encyclopedia of Planetary Sciences (Berlin: Springer)

[15] Roberts P H and King E M 2013 Rep. Prog. Phys. 76096801

[16] Mininni P D and Pouquet A 2010 Phys. Fluids 22035105

[17] Mininni P D, Rosenberg D and Pouquet A 2012 J. Fluid Mech. 699 263-79

[18] Teitelbaum T and Mininni P D 2012 Phys. Rev. Lett. 103014501

[19] Galtier S 2003 Phys. Rev. E 68015301

[20] Galtier S, Nazarenko S and Newell A C 2001 Nonlinear Process. Geophys. 8 141-50

[21] Meyrand R, Kiyani K and Galtier S 2015 J. Fluid Mech. 770 R1

[22] Iroshnikov P S 1964 Sov. Astron. 7 566-71

[23] Kraichnan R H 1965 Phys. Fluids 8 1385-7

[24] Meyrand R, Galtier S and Kiyani K 2016 Phys. Rev. Lett. 116105002

[25] Meyrand R, Kiyani K, Gürcan O D and Galtier S 2018 Phys. Rev. X 8031066

[26] Nazarenko S and Schekochichin A 2011 J. Fluid Mech. 677134

[27] Kolmogorov A N 1941 Dokl. Akad. Nauk. SSSR 30 301-5

[28] Gómez D O, Mininni P D and Dmitruk P 2005 Phys. Scr. T116 123

[29] Mininni P D and Pouquet A 2007 Phys. Rev. Lett. 99254502

[30] Mininni P D, Rosenberg D, Reddy R and Pouquet A 2011 Parallel Comput. 37 316-26

[31] Nazarenko S and Onorato M 2006 Physica D 219 1-12

[32] Nazarenko S and Onorato M 2007 J. Low Temp. Phys. 146 31-46

[33] Boué L, Dasgupta R, Laurie J, L'vov V, Nazarenko S and Procaccia I 2011 Phys. Rev. B 84064516

[34] Leoni P C, Cobelli P J and Mininni P D 2015 Eur. Phys. J. E 38136

[35] TenBarge J M and Howes G G 2011 Phys. Plasmas 195 